\title{
Spectral estimation of the percolation transition in clustered networks
}

\author{
Pan Zhang* ${ }^{*}$ \\ CAS key Laboratory of Theoretical Physics, Institute of Theoretical Physics, \\ Chinese Academy of Sciences, Beijing 100190, China.
}

\begin{abstract}
There have been several spectral bounds for the percolation transition in networks, using spectrum of matrices associated with the network such as the adjacency matrix and the non-backtracking matrix. However they are far from being tight when the network is sparse and displays clustering or transitivity, which is represented by existence of short loops e.g. triangles. In this work, for the bond percolation, we first propose a message passing algorithm for calculating size of percolating clusters considering effects of triangles, then relate the percolation transition to the leading eigenvalue of a matrix that we name the triangle-non-backtracking matrix, by analyzing stability of the message passing equations. We establish that our method gives a tighter lower-bound to the bond percolation transition than previous spectral bounds, and it becomes exact for an infinite network with no loops longer than 3. We evaluate numerically our methods on synthetic and real-world networks, and discuss further generalizations of our approach to include higher-order sub-structures.
\end{abstract}

\section{INTRODUCTION}

Percolation in networks is a well-known process that has been studied extensively in many fields of science. It found numerous applications in physics, networks, material science and social science [1 9 . In this work we study the bond percolation on an arbitrary undirected network, where each edge is open with probability $p$ and close with probability $1-p$. In the $n \rightarrow \infty$ limit, depending on $p$, in the network there could be one giant cluster that contains a finite fraction of nodes in addition to many small clusters with vanishing fraction of nodes, or no giant cluster at all. The transition value $p^{*}$ determines the smallest value of $p$ that with high probability the giant cluster emerges.

There have been lots of analytical studies of percolation transition on synthetic networks, such as random graphs 2, 10 12, generalization of random graphs with triangles and cliques 13 17] etc. However most of those studies are specific for ensemble of networks in the $n \rightarrow \infty$ limit, rather than a given (synthetic or real-world) network. Recently there are studies on approximating the percolation transition for a given real-world network using spectral properties of matrices that associated with the network. Here we use "approximate" because the percolation transition is legally defined only for infinite networks, where an infinite giant cluster could appear. On finite networks, one usually identifies the percolation transition by the point where the second-largest cluster has the greatest size, which converges to the percolation threshold when system size goes to infinity.

This can be done by running numerous direct simulations to compute the size of the largest connected component on each realization of random occupations of edges, then take the ensemble average. However this is time-consuming. In $[18$ it is proposed to use inverse spectral radius of the network, that is inverse of the leading eigenvalue of the network's adjacency matrix $A$, as an estimate of the percolation transition. This estimate is accurate when the network is dense, but heavily underestimates the percolation transition on sparse networks. In recent work [19, 20, the authors proposed to approximate percolation transition on sparse networks using inverse of leading eigenvalue of the non-backtracking matrix [21 23], which is defined on directed edges of the network. In addition to computational speed, another motivation of using spectral methods for the percolation is that it provides more information on the percolation than the size of clusters in a compact way, which can be used to organize efficient algorithms for applications based on percolation, such as the network dismantling problem [24, 25.

The idea behind the use of the non-backtracking matrix comes from the linearization of belief propagation (BP) [26] equations around a factorized fixed point. Since the belief propagation assumes the conditional independence, it is exact when effects of loops in the network can be neglected, and is a good approximation in real-world networks. Authors in [19, 20] have shown that the estimation of the percolation transition using the non-backtracking matrix is a lower-bound to the true percolation transition on an infinite undirected network, and is exact when the network is an infinite tree. In 20] by comparing the estimate to the direct simulation of percolation processes on real-world networks, the authors also showed that the estimate is a better approximation than the inverse for spectral radius for real-world networks, since most of the real-world networks are sparse.

*Electronic address: panzhang@itp.ac.cn 
We note here that in addition to sparsity, another characteristic of real-world networks is clustering, or transitivity, which is represented by existence of short loops like triangles in the network. However the estimate of percolation transition using the non-backtracking matrix assumes that the network is locally tree like, thus ignores effects of short loops. In this work we address the problem of how to incorporate the loops, especially, triangles, in estimating the percolation transition.

Given a general network $G$ with $n$ nodes and $m$ edges, we can decompose the graph into set of triangles $\mathbb{T}$ and set of single edges $\mathbb{E}$ that do not share common edges, which we term the $\{\mathbb{T}, \mathbb{E}\}$ decomposition. It is natural to define a factor graph composed of $\mathbb{T}$ and $\mathbb{E}$ where triangles and single edges are treated as two different kinds of factors, that is a factor graph having both two-body interactions and three-body interactions, as in the classic $2+p$ Satisfiability problem [27. An example of the decomposition is given in Fig. 1 (a). In the figure the graph contains 11 nodes, 3 triangles and 4 single edges, hence in the factor graph there are 11 variables and 7 factors. Then we apply Belief Propagation (BP) algorithm on this factor graph to compute the marginal probability of each node being in the percolation cluster. Then the percolation transition can be estimated using the marginals which can be simplified to an eigenvector problem of a matrix, that we call Triangle-Non-Backtracking matrix.

When the network contains no loops longer than 3, as shown in the Fig. 1 , the $\{\mathbb{T}, \mathbb{E}\}$ decomposition of the graph is unique, and marginals given by $\mathrm{BP}$ is exact which results to an exact estimate of percolation transition. However if network do contains loops longer than 3 , the $\{\mathbb{T}, \mathbb{E}\}$ decomposition is not unique. An example of choosing a $\{\mathbb{T}, \mathbb{E}\}$ decomposition is illustrated in Fig. 1 where the graph has two triangles sharing an edge, thus there are two ways to decompose the graph. In the figure only one way to do the decomposition is shown: putting the (red) upper triangle as a triangle and putting blue edges as single edges. In this paper we do not address the problem of selecting the optimal $\{\mathbb{T}, \mathbb{E}\}$ decomposition. Instead we note that for a randomly selected $\{\mathbb{T}, \mathbb{E}\}$ decomposition, our approach gives a provable better estimate of the percolation transition than the existing spectral bounds.

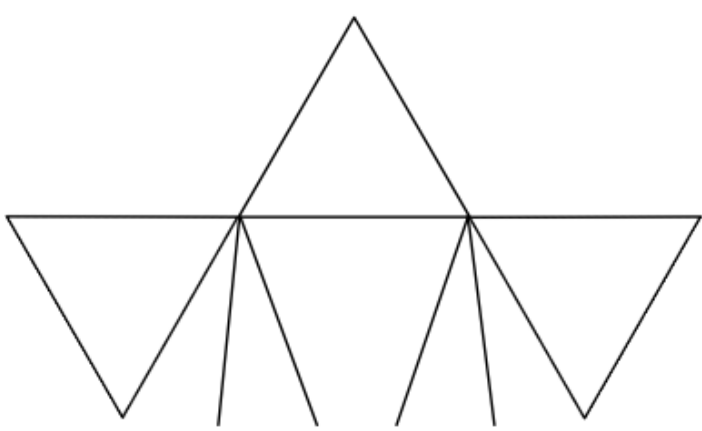

(a)

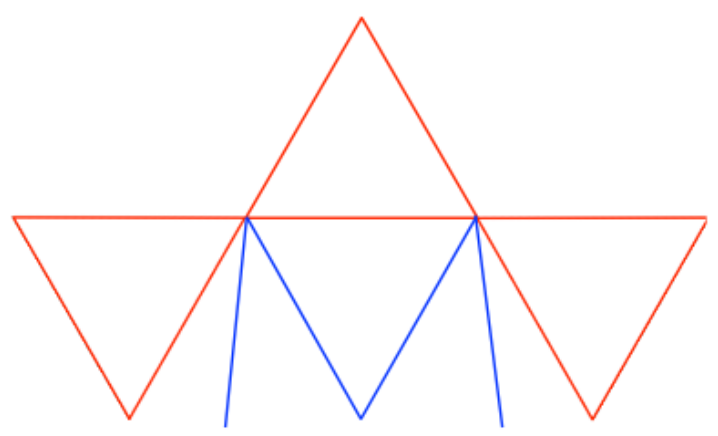

(b)

FIG. 1: (Color online) (a) A graph with no loop longer than 3. (b) One way to decompose a graph containing loops longer than 3 to triangles (red) and single edges (blue).

\section{MESSAGE PASSING CONSIDERING TRIANGLES}

In this paper we will address the bond percolation problem, the site percolation problem can be addressed using the analogous technique. For the bond percolation, each edge is open with probability $p$ and is close with probability $1-p$. We are interested in size of cluster that each node belongs to $\left\{s_{i}\right\}: i \in[1, n]$ (where $s_{i}$ denotes the size of cluster that node $i$ belongs to, $n$ denotes the number of nodes). Since each realization of open-close configuration of edges is a random variable, we can not predict $\left\{s_{i}\right\}$ for each realization. Instead we are interested in the probability of node $i$ being in cluster of size $s, \psi_{s}^{i}$, the probability defined on all realizations of the open-close states of edges. Particularly we are interested in the size of the giant cluster $\hat{s}_{1}(p)$, which is an increasing function of $p$. For an infinite network, the percolation transition $p_{c}$ is defined at the critical point where $\hat{s}_{1}(p)$ changes from 0 to a finite value, indicating that the giant percolation cluster fills a non-vanishing fraction of nodes in the network. For a real-world network which has a finite size, we are also interested in the size of the second largest percolation cluster $\hat{s}_{2}(p)$, as we can define $p^{*}$ at the point where the $\hat{s}_{2}(p)$ begins decreasing as $p$ increases.

We start with consistent equations for the size of finite clusters that a node $i$ belongs to. When the graph has no loops longer than 3, we can effectively treat the factor graph as a tree rooted at $i$ and has two kinds of descendants: through singles edges and through triangles. Clearly different descendants do not share common nodes, thus size of the cluster that node $i$ belongs to, $s_{i}$, is sum of cluster sizes of reachable (i.e. connected 
by a open edge) descendants. Then the probability that node $i$ belongs to a cluster with size $s$ can be written as $\psi_{s_{i}}^{i}=\sum_{\left\{s_{i^{\prime}} i^{\prime} \in \partial^{-} i\right\}} \sum_{\left\{s_{a}: a \in \partial^{\Delta} i\right\}} \delta\left(s_{i}-1, \sum_{i^{\prime}} s_{i^{\prime}}+\sum_{a} s_{a}\right) \operatorname{Prob}\left(\left\{s_{i^{\prime}}\right\},\left\{s_{a}\right\}\right)$, where $\delta(a, b)$ is the Kronecker delta function, $\partial^{-} i$ denotes the set of neighbors of node $i$ through a single edge, $\partial^{\Delta} i$ denotes the set of triangles connected to node $i$, and Prob $\left(\left\{s_{i^{\prime}}\right\},\left\{s_{a}\right\}\right)$ denotes the joint probability of sizes reachable through triangles and single edges respectively. As the factor graph is a tree, the joint probability of sizes can be written in a factorized form, which results to

$$
\psi_{s}^{i}=\sum_{\left\{s_{i^{\prime}}: i^{\prime} \in \partial^{-} i\right\}} \sum_{\left\{s_{a}: a \in \partial^{\Delta} i\right\}} \delta\left(s_{i}-1, \sum_{i^{\prime}} s_{i^{\prime}}+\sum_{a} s_{a}\right) \prod_{i^{\prime} \in \partial^{-} i} \psi_{s_{i^{\prime}}}^{i^{\prime} \rightarrow i} \prod_{a \in \partial^{\Delta} i} \psi_{s_{a}}^{a \rightarrow i} .
$$

Here $\psi_{s_{i^{\prime}}}^{i^{\prime} \rightarrow i}$ denotes the probability that size of cluster that node $i^{\prime}$ is reachable from $i$ through a single edge, and $\psi_{s_{a}}^{a \rightarrow i}$ is the probability that triangle $a$ belongs to a cluster with size $s_{a}$ that is reachable from node $i$.

If a single edge is close (with probability $1-p$ ) the size of cluster that reachable through $i$ is clearly 0 , otherwise it must be a finite value. Thus cavity probabilities $\psi_{i \rightarrow l}^{s_{i}}$ can be computed as

$$
\psi_{s_{i}}^{i \rightarrow l}=(1-p) \delta\left(s_{i}, 0\right)+p \sum_{\left\{s_{a}: a \in \partial^{\Delta} i\right\}} \sum_{\left\{s_{i^{\prime}}: i^{\prime} \in \partial^{-} \backslash \backslash \backslash\right\}} \delta\left(s_{i}-1, \sum_{i^{\prime}} s_{i^{\prime}}+\sum_{a} s_{a}\right) \prod_{i^{\prime}} \psi_{s_{i^{\prime}}}^{i^{\prime} \rightarrow i} \prod_{a} \psi_{s_{a}}^{a \rightarrow i} .
$$

Messages sent from triangle $a=(i, j, k)$ to a node $i, \psi_{s_{a}}^{a \rightarrow i}$, are more complex, as the probability depends on whether edges $(i, j),(j, k)$ and $(i, k)$ are open or close: If both $(i, j)$ and $(i, k)$ are close, with probability $(1-p)^{2}$, the number of nodes reachable from node $i$ through triangle $a, s_{a}$ is 0 ; If both $(i, j)$ and $(i, k)$ are open, then node $j$ and $k$ are both reachable from node $i$, we can see that $s_{a}-1$ should equal to the number of nodes in clusters that both $j$ and $k$ belong to; If only one of two neighbors, say $j$, is reachable from $i$, then $s_{a}-1$ should be the number of nodes in the cluster that $j$ belongs to. Therefore we have the following equation for the probability of number of nodes reachable from $i$ through triangle $a=(i, j, k)$,

$$
\begin{aligned}
\psi_{s_{a}}^{a \rightarrow i} & =(1-p)^{2} \delta\left(s_{a}, 0\right) \\
& +p(1-p)^{2} \sum_{\left\{s_{j^{\prime}}\right\}: j^{\prime} \in \partial^{-}} \sum_{\left\{s_{b}\right\}: b \in \partial^{\Delta} j \backslash a} \delta\left(s_{a}-1, \sum_{j^{\prime}} s_{j^{\prime}}+\sum_{b} s_{b}\right) \prod_{j^{\prime}} \psi_{s_{j^{\prime}}}^{j^{\prime} \rightarrow j} \prod_{b} \psi_{s_{b}}^{b \rightarrow j} \\
& \left.+p(1-p)^{2} \sum_{\left\{s_{k^{\prime}}\right\}: k^{\prime} \in \partial^{-} k} \sum_{\left\{s_{f}:\right\} f \in \partial^{\Delta} k \backslash a} \delta\left(s_{a}-1, \sum_{k^{\prime}} s_{k^{\prime}}+\sum_{f} s_{f}\right)\right) \prod_{k^{\prime}} \psi_{s_{k^{\prime}}}^{k^{\prime} \rightarrow k} \prod_{f} \psi_{s_{f}}^{f \rightarrow k} \\
& +\left(3 p^{2}-2 p^{3}\right) \sum_{\left\{s_{j^{\prime}}\right\}: j^{\prime} \in \partial^{-}} \sum_{\left\{s_{b}\right\}: b \in \partial j^{\Delta} \backslash a} \sum_{\left\{s_{k^{\prime}}\right\}: k^{\prime} \in \partial^{-}} \sum_{\left\{s_{f}\right\} f \in \partial k^{\Delta} \backslash a} \prod_{j^{\prime}} \psi_{s_{j^{\prime}}}^{j^{\prime} \rightarrow j} \prod_{b} \psi_{s_{b}}^{b \rightarrow j} \\
& \cdot \prod_{k^{\prime}} \psi_{s_{k^{\prime}}}^{k^{\prime} \rightarrow k} \prod_{f} \psi_{s_{f}}^{f \rightarrow k} \delta\left(s_{a}-1, \sum_{j^{\prime}} s_{j^{\prime}}+\sum_{b} s_{b}+\sum_{k^{\prime}} s_{k^{\prime}}+\sum_{f} s_{f}\right)
\end{aligned}
$$

We need to notice that the cluster size in the last equation must be finite, as only if $\left\{s_{j}\right\}$ and $\left\{s_{a}\right\}$ are finite values, will the equality $s_{i}-1=\sum_{\left\{s_{j}: j \in \partial^{-} i\right\}}+\sum_{\left\{s_{a}: a \in \partial^{\Delta} i\right\}}$ make sense. Otherwise if cluster size of at least one of $i$ 's neighbors is $\infty$, then $s_{i}$ must be $\infty$ regardless of the value of other cluster sizes.

We can see that equations above are difficult to solve, because the number of states is large. So instead of trying to determine $\psi_{s_{i}}^{i}$, we introduce the total probability that a node belongs to finite clusters

$$
\eta^{i}=\sum_{s_{i}=1}^{\infty} \psi_{s_{i}}^{i}=\prod_{i^{\prime} \in \partial^{-} i} \eta^{i^{\prime} \rightarrow i} \prod_{a \in \partial^{\Delta} i} \eta^{a \rightarrow i}
$$

where $\eta^{i^{\prime} \rightarrow i}$ and $\eta^{a \rightarrow i}$ are probabilities that $i^{\prime}$ or $a$ belongs to finite clusters, and will be introduced later. Eq. (2) actually has a simple meaning: A node belongs to a finite cluster only when all its reachable neighbors belong to finite clusters. Then notice that the probability of a node $i$ belongs to an infinite (percolating) cluster is simply $1-\eta^{i}$. In the above equation the joint probability of its reachable neighbors belonging to finite clusters has the factorized form because we assumed that the factor graph is locally-tree-like, and different neighbors in different branches of the tree 
rooted at $i$ have no common children. The probabilities of neighbors belonging to finite clusters can be computed in a similar way for both along an edge $i \rightarrow j$ and from a triangle $a=(i, j, k)$ to one of its end-point $i$, as illustrated in Fig. 2

$$
\begin{aligned}
\eta^{i \rightarrow j} & =\sum_{s=0}^{\infty} \psi_{s}^{i \rightarrow j}=1-p+p \prod_{i^{\prime} \in \partial i^{-} \backslash j} \eta^{i^{\prime} \rightarrow i} \prod_{a \in \partial^{\Delta} i} \eta^{a \rightarrow i} \\
\eta^{a \rightarrow i} & =\sum_{s=0}^{\infty} \psi_{s}^{a \rightarrow i}=\left(3 p^{2}-2 p^{3}\right) \prod_{j^{\prime} \in \partial^{-} j} \eta^{j^{\prime} \rightarrow j} \prod_{b \in \partial^{\Delta} j} \eta^{b \rightarrow j} \prod_{k^{\prime} \in \partial^{-} k} \eta^{k^{\prime} \rightarrow k} \prod_{f \in \partial^{\Delta} k} \eta^{f \rightarrow k} \\
& +(1-p)^{2}+p(1-p)^{2}\left(\prod_{j^{\prime} \in \partial^{-} j} \eta^{j^{\prime} \rightarrow j} \prod_{b \in \partial^{\Delta} j} \eta^{b \rightarrow j}+\prod_{k^{\prime} \in \partial^{-} k} \eta^{k^{\prime} \rightarrow k} \prod_{f \in \partial^{\Delta} k} \eta^{f \rightarrow k}\right) .
\end{aligned}
$$

Above equations are belief propagation equations on the factor graph associated with a $\{\mathbb{E}, \mathbb{T}\}$ decomposition of the network. We can initialize BP messages randomly then update messages using BP iterative equations in random sequential order. After messages converge, the probability of each node being in finite clusters can be computed using Eq. 22). We can also compute the expectation of fraction of nodes in the percolation cluster is given by $\tilde{s}=\frac{1}{n} \sum_{i=1}^{n}\left(1-\eta^{i}\right)$. We note that our messages $\eta^{j \rightarrow i}$ and $\eta^{a \rightarrow i}$ correspond to a special case of generating functions used in [20]. Other quantities, e.g. size of the non-percolating cluster that a node belongs to, can be computed using the generating functions technique [20], but will not be shown here. It is straightforward to see that if for every node $i$ such that $\eta^{i}=1$ we have $\tilde{s}=0$. This means that every node belongs to finite clusters hence there is no percolating cluster occupying finite fraction of nodes. When $\tilde{s}>0$, i.e., for some $i, \eta^{i}$ deviates from 1 , then obviously system has a percolation cluster. Thus where $\eta^{i}$ deviate from 1 tells us the position of the estimated percolation transition.

Notice that BP equations on the factor graph are exact only when the graph contains no loops that are longer than 3. On general graphs which do have loops longer than 3 , although the fact that finite-reachable nodes from neighbors leads to a finite-size cluster still holds, we can not express the joint probability that neighbors belonging to finite clusters as a factorized form. Instead, for $i^{\prime} \in \partial^{-} i$ and $a \in \partial^{\Delta} i$ we need to use

$$
\eta^{i}=\operatorname{Prob}\left(\left\{s_{i^{\prime}}\right\},\left\{s_{a}\right\} \text { are finite }\right),
$$

and probabilities in Eq. (3) (4) need to be reformulated as

$$
\begin{aligned}
\eta^{i \rightarrow j} & =1-p+p \cdot \operatorname{Prob}\left(\left\{s_{i^{\prime}}\right\},\left\{s_{a}\right\} \text { are finite }\right) \\
\eta^{a \rightarrow i} & =(1-p)^{2}+p(1-p)^{2} \cdot\left[\operatorname{Prob}\left(\left\{s_{j^{\prime}}\right\},\left\{s_{b}\right\} \text { are finite }\right)+\operatorname{Prob}\left(\left\{s_{k^{\prime}}\right\},\left\{s_{f}\right\} \text { are finite }\right)\right] \\
& +\left(3 p^{2}-2 p^{3}\right) \cdot \operatorname{Prob}\left(\left\{s_{j^{\prime}}\right\},\left\{s_{b}\right\},\left\{s_{k^{\prime}}\right\},\left\{s_{f}\right\} \text { are finite }\right) .
\end{aligned}
$$

Since above probabilities are positively correlated when there are loops longer than 3, generally we have $\operatorname{Prob}\left(\left\{s_{i^{\prime}}\right\},\left\{s_{a}\right\}\right.$ are finite $) \geq \prod_{i^{\prime}} \eta^{i^{\prime} \rightarrow i} \prod_{a} \eta^{a \rightarrow i}$. As a consequence, when BP equations converge, the obtained $\eta^{i}$ is only a lower-bound to the true probability of node belonging to finite clusters, and deviates from 1 earlier than the true percolation transition as $p$ increases from a small value. Thus percolation transition estimated using BP is only a lower-bound to the true percolation transition on an infinite size network.

\section{THE TRIANGLE-NON-BACKTRACKING MATRIX}

We observe that $\eta^{i}=\eta^{i \rightarrow j}=\eta^{a \rightarrow i}=1$ is always a fixed-point of BP Eqs.(3) (4). This fixed-point means that all nodes belong to non-percolation clusters so the size of largest cluster is finite and the fraction of nodes that the percolation cluster occupies is 0 . We call this fixed-point the factorized fixed-point. It is easy to check that at $p=0$ the factorized fixed-point is the only fixed-point; with $p$ small the factorized fixed-point is locally stable to perturbations; when $p$ is large, the factorized fixed-point could be unstable during iteration to an infinitesimal perturbations, and $\mathrm{BP}$ converges to another fixed-point with a finite fraction of nodes occupied by the giant cluster. Therefore the critical point $p^{*}$ where the factorized fixed-point becomes unstable is our estimate of the percolation transition, and it can be studied by analyzing the stability of the factorized fixed-point.

We expand BP messages at the factorized fixed-point:

$$
\eta^{i \rightarrow j}=1-\epsilon^{i \rightarrow j} \quad \eta^{a \rightarrow i}=1-\epsilon^{a \rightarrow i},
$$




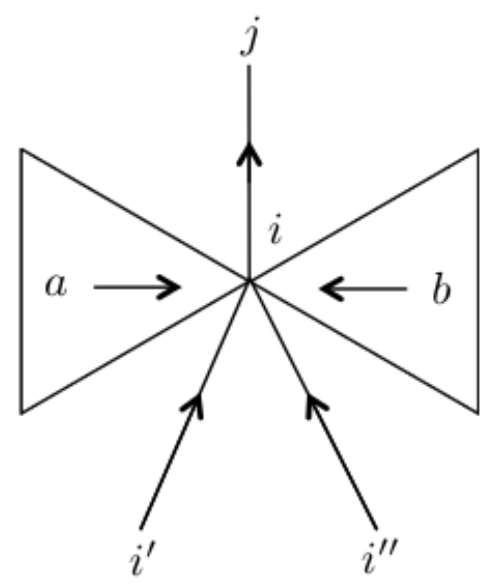

(a)

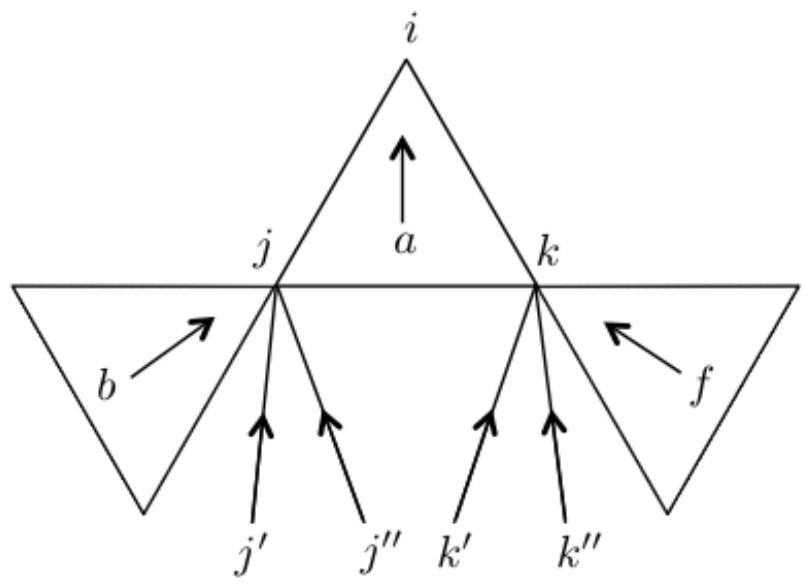

(b)

FIG. 2: (a): Illustration of the computation of BP messages along a single edge $i \rightarrow j$, using messages from triangles and single edges to $i$, except $j \rightarrow i$. (b): Illustration of computation of messages from a triangle $a$ to node $i$, as a function of messages from other triangles and from single edges.

then the iterative equations for the deviation of messages to the factorized fixed-point, along a directed edge $i \rightarrow j$ and from triangle $a=(i, j, k)$ to one of its end-point $i$, can be written as

$$
\begin{aligned}
\epsilon^{i \rightarrow j} & :=p \sum_{i^{\prime} \in \partial i^{-} \backslash j} \epsilon^{i^{\prime} \rightarrow i}+p \sum_{a \in \partial^{\Delta} i} \epsilon^{a \rightarrow i} \\
\epsilon^{a \rightarrow i} & :=q \sum_{j^{\prime} \in \partial j^{-}} \epsilon^{j^{\prime} \rightarrow j}+q \sum_{b \in \partial^{\Delta} j \backslash a} \epsilon^{b \rightarrow j}+q \sum_{k^{\prime} \in \partial k^{-}} \epsilon^{k^{\prime} \rightarrow k}+q \sum_{f \in \partial^{\Delta} k \backslash a} \epsilon^{f \rightarrow k},
\end{aligned}
$$

with $q=p+p^{2}-p^{3}$. Above equations can be rewritten as a matrix form $\lambda \epsilon=C \epsilon$, where $\lambda$ is an eigenvalue and $C$ is the matrix that we refer as Triangle-Non-Backtracking (TNB) matrix, whose elements $x \rightarrow i$ and $z \rightarrow w$ are messages along directed single edges or from a triangle to one of its end-point:

$$
C_{x \rightarrow i, z \rightarrow w}=p \mathbb{1}_{x \notin \mathbb{T}} \delta(x, w)(1-\delta(z, i))+q \mathbb{1}_{x \in \mathbb{T}} \mathbb{1}_{w \in x \backslash i} \mathbb{1}_{z \notin x} .
$$

Here $\mathbb{1}$ is the indicator function. We can see that analogous to the non-backtracking matrix, the TNB matrix represents the non-backtracking walks in the network, but avoids also the backtrackings along (weighted) edges of triangles.

We are interested in the largest eigenvalue $\lambda_{C}$ and the corresponding leading eigenvector $\epsilon_{1}$. Eq. (8) offers a method for its evaluation. As every element of matrix $C$ is non-negative, Perron-Frobenius theorem applies and we can conclude that the $\lambda_{C} \geq 0$ and elements of $\epsilon_{1}$ are non-negative. $\lambda_{C}$ is actually a stability parameter for BP factorized fixed-point: When $\lambda_{C}<1$, the factorized fixed-point is stable, system has no percolating cluster; when $\lambda_{C}>1$, system has a percolating cluster occupying finite fraction of nodes. So $\lambda_{C}=1$ gives a critical percolation probability $p_{C}^{*}$ (note that $\lambda_{C}$ is an increasing function of $p$ ). Furthermore, we establish in the following lemma that on an arbitrary graph, $\lambda_{C}$ is bounded above by $p \lambda_{B}$ when $p \lambda_{B} \leq 1$, where $\lambda_{B}$ is the leading eigenvalue of the non-backtracking matrix $B$. The proof to the lemma can be found in the appendices.

Lemma 1 Let $\lambda_{B}$ be the largest eigenvalue of the non-backtracking matrix, $\lambda_{C}$ be the largest eigenvalue of the trianglenon-backtracking matrix associated with any $\{\mathbb{T}, \mathbb{E}\}$ decomposition of the network. Then for any undirected graph, we have $p \lambda_{B} \geq \lambda_{C}$ when $p \lambda_{B} \leq 1$.

The above lemma says that below the percolation transition given by $p^{*} \lambda_{B}=1, \lambda_{C}$ never excesses 1 . With the fact that $\lambda_{C}$ is an increasing function of $p$, we conclude that the critical probability that makes $\lambda_{C}=1$ is bounded below by $p^{*} \lambda_{B}$. Also by making use of the theorem in [19, 20] that the leading eigenvalue of the adjacency matrix, $\lambda_{A}$ is strictly larger than $\lambda_{B}$, we prove the following theorem.

Theorem 2 Let $p_{C}^{*}$ satisfy $\lambda_{C}=1$ for the triangle-non-backtracking matrix $C$ associated with an arbitrary $\{\mathbb{T}, \mathbb{E}\}$ decomposition of the graph, let $p_{B}^{*}=\frac{1}{\lambda_{B}}, p_{A}^{*}=\frac{1}{\lambda_{A}}$, then on an arbitrary undirected graph, $p_{A}^{*}<p_{B}^{*} \leq p_{C}^{*}$. 
So, in general, the percolation transition making the leading eigenvalue of the triangle-non-backtracking matrix equal 1 is a tighter lower bound to the true percolation transition than the lower-bound given by the inverse of the leading eigenvalue of the non-backtracking matrix and the adjacency matrix.

If we use $n^{-}$to indicate the number of single edges and $n^{\Delta}$ indicate number of triangles, then the TNB matrix Eq. A4 has size $2 n^{-}+3 n^{\Delta}$, which becomes larger when network becomes denser. Fortunately we see that the non-trivial part of spectrum of $\mathrm{C}$ can be obtained from a matrix $C^{\prime}$ (as defined in Definition 3) with size $4 n \times 4 n$ — which does not increase with number of edges. This statement is established in Theorem 4 and is proved in the appendices.

Definition 3 Define matrix $C^{\prime}$ that associated with a $\{\mathbb{E}, \mathbb{T}\}$ decomposition as

$$
C^{\prime}=\left(\begin{array}{cccc}
p \mathbb{A}^{-} & -p \mathbb{I} & p \mathbb{A}^{-} & 0 \\
p\left(\mathbb{D}^{-}-\mathbb{I}\right) & 0 & p \mathbb{D}^{-} & 0 \\
q \mathbb{A}^{\Delta} & 0 & q \mathbb{A}^{\Delta} & -q \mathbb{I} \\
2 q \mathbb{D}^{\Delta}+q \mathbb{A}^{\Delta} & 0 & 2 q\left(\mathbb{D}^{\Delta}-\mathbb{I}\right)+q \mathbb{A}^{\Delta} & -q \mathbb{I}
\end{array}\right) .
$$

Here $\mathbb{I}$ denotes the $n$-dimensional identity matrix, $\mathbb{A}^{-}$is the adjacency matrix of nodes connected by single edges; $\mathbb{D}^{-}$ is the diagonal matrix of single-edge-degrees; $\mathbb{A}^{\Delta}$ is the adjacency matrix of nodes connected by triangles; $\mathbb{D}^{\Delta}$ is the diagonal matrix of triangle-degrees. That is

$$
\begin{array}{rlrl}
\mathbb{A}_{i, j}^{-} & = \begin{cases}1, \text { if }(i, j) \in \mathbb{E} \\
0, \text { other wise }\end{cases} & \mathbb{A}_{i, j}^{\Delta}=\left\{\begin{array}{l}
1, \text { if } \exists(i, j, l) \in \mathbb{T} \\
0, \text { other wise }
\end{array}\right. \\
\mathbb{D}_{i, i}^{-}=\sum_{k} \mathbb{A}_{i k}^{-} & \mathbb{D}_{i, i}^{\Delta}=\frac{1}{2} \sum_{k} \mathbb{A}_{i k}^{\Delta} .
\end{array}
$$

Theorem 4 If $C$ and $C^{\prime}$ are associated with the same $\{\mathbb{T}, \mathbb{E}\}$ decomposition of an arbitrary graph, then non-zero eigenvalues of two matrices are identical.

Thus working with this $4 n$ dimensional matrix can significantly reduce the computational complexity of finding the leading eigenvalue of matrix $C$. Obviously when the network has no loops longer than 3 , that is the factor graph treating both single edges and triangles as factors is a tree, $p_{C}^{*}$ is exact. It is interesting to see that when the factor graph is a finite tree, $\lambda_{C}=0$. This is because when we can find the leaves in the factor graph, the elements in the matrix $C$ which corresponds to the single edges connected to the leaves must be 0 . The 0 messages will be propagated further to all the network, resulting to $\lambda_{C}=0$. This do makes sense in the finite-size networks, as there could not be infinite-size cluster or percolation transition defined in the system. Also note that when the network is infinite, where it is not possible to define the concept of a leave, $\lambda_{C}>0$ due to proper boundary conditions. At the thermodynamic limit the (asymptotic) exactness of our method does not require the factor graph to be a perfect tree. Instead, when the fraction of short loops in the factor graph is negligible, in other words when correlations decays fast enough [41] our method would be asymptotically exact. Similar arguments on the asymptotically exactness have been used in [20], as well as in the context of community detection in networks [42].

When the infinite network do have loops longer than 3, the resulting factor graph is no longer a tree, then our estimate $p_{C}^{*}$ on any $\{\mathbb{T}, \mathbb{E}\}$ decomposition of single edges and triangles is a lower bound for the true percolation transition, and is tighter than the inverse of the non-backtracking matrix.

\section{EXPERIMENTAL EVALUATION}

In this section, to evaluate our message passing algorithm, as well as the estimate of percolation transition using the TNB matrix, we conduct direct simulations of percolation processes on both synthetic and real-world networks and compare the average size of the giant cluster and the percolation transition with our methods.

\section{A. Synthetic networks}

For synthetic networks we use the Clustered Random Graph (CRG) model proposed in 28]. In the CRG model, the standard random graph models are generalized to incorporate triangles. The model has a tunable clustering coefficient by specifying both number of triangles and number of single edges then distributing these edges and triangles into 
networks in a random and uncorrelated way. In our simulations we use parameter $\rho$ to specify number of edges belonging to triangles.

Since we need to test the performance of our message passing algorithm and the threshold given by the trianglenon-backtracking matrix, we conduct simulations on generated finite networks which do have loops longer than 3 . So we first choose a (random) decomposition of the generated network into single edges and triangles randomly, then run our BP algorithm and triangle-non-backtracking matrix on the resulting graph. We compare the size of the giant cluster in simulation with that computed using our method, and the state-of-art method for sparse networks proposed in [20] which does not incorporate effects of triangles. The comparison is shown in Fig. 3 for CRG networks with a Poisson degree distribution. From the figure we can see that the size of the giant cluster given by our method is in agreement with the simulation, while the message passing without considering triangles as proposed in 20] overestimates the average size of the giant cluster, hence underestimates the percolation transition. In the inset of the figure we plot the percolation transition estimated using the adjacency matrix, the non-backtracking matrix, and the triangle-non-backtracking matrix, as well as the second largest component size, which displays a peak at $p^{*}$ which can be seen as an estimate of percolation transitions in a finite network. As the figure shows, the threshold given by the triangle-non-backtracking matrix is much closer to $p^{*}$ than the adjacency matrix and the non-backtracking matrix. All three estimates are smaller than $\rho^{*}$, which is consistent with the argument that they are all lower bounds for the true percolation transition on an infinite network.

In the CRG model, the exact properties of bond percolation with $n \rightarrow \infty$ has already been calculated [28]. So we only need to test whether the thresholds given by the triangle-non-backtracking matrix on large network agree with the $n \rightarrow \infty$ theory in [28. In Fig. 3 we compare thresholds given by the adjacency matrix $A$, the non-backtracking matrix $B$ and the triangle-non-backtracking matrix $C$ on large CRG networks with $n=10^{6}$ nodes, average degree $c=3$ and varying fraction of edges belonging to triangles $\rho$, with the theoretical results for $n \rightarrow \infty$ in 28. As the figure shows, the threshold given by $C$ agrees very well with the $n \rightarrow \infty$ theory while thresholds given by $B$ and $A$ are much worse.

\section{B. Real world networks}

In Table $\mathrm{I}$ we compare percolation transitions estimated using different matrices with simulations, on several networks with size ranging from 34 nodes to $10^{5}$ nodes. The first 10 networks in the table are real-world networks with references given in the table. The last two networks are synthetic networks with community structures, they are generated by the Stochastic Block Model (SBM) and its variant Triangular Stochastic Block Model (TSBM [unpublished]) which generates random graphs with both local (triangles) and global clusters (communities). Since there is no real phase transition in finite-size networks, for simulation results $p_{\text {simu }}^{*}$ are taken at the point where the second largest cluster is greatest. The number in the parentheses indicate the error on the last digit. The columns $p_{A}^{*}, p_{B}^{*}$, and $p_{C}^{*}$ refer to the percolation transition estimated using the leading eigenvalue of the adjacency matrix, non-backtracking matrix and the triangle-non-backtracking matrix respectively. We can see from the table that the errors of $p_{C}^{*}$ (number in parentheses) over different $\{\mathbb{T}, \mathbb{E}\}$ decompositions are quite small. In agree with our theoretical justifications, our results shows that thresholds estimated using the eigenvalues of triangle-non-backtracking matrix are always lower than the simulation results, and are higher than those obtained using the non-backtracking matrices and adjacency matrices. For some networks such as the Karate club and TSBM networks, the improvement of $p_{C}^{*}$ over $p_{B}^{*}$ is quite large, while on networks which contains almost no triangles (such as the SBM network), $p_{C}^{*}$ is very close to $p_{B}^{*}$.

For the SBM network, we can see a little difference between $\rho_{B}^{*}$ and $\rho_{C}^{*}$, which is clearly due to existing of few triangles (6 in this instance of SBM network). There are also a significant difference between $\rho_{\text {simu }}^{*}$ and $\rho_{B}^{*}$. We claim it should come from the finite size effect of using the point where the second-largest cluster is greatest as the estimate to the phase transition. With system size increases, first we have checked that both $\rho_{B}^{*}$ and $\rho_{C}^{*}$ converge to 0.333 , as the average excess degree (which equals to the average degree in with Poisson degree distribution) is 3.0; then we confirm that $p_{\text {simu }}^{*}$ decreases with $n$ increasing. For example when network size increases to $5 \times 10^{5}, p_{\text {simu }}^{*}$ decreases to $0.338(1)$. Thus we expect $\rho_{B}^{*}, \rho_{C}^{*}$, and $\rho_{\text {simu }}^{*}$ coincide with network size $n \rightarrow \infty$ for SBM network, and expect $\rho_{C}^{*}$ and $\rho_{\text {simu }}^{*}$ coincide for the same reason.

\section{CONCLUSIONS AND DISCUSSIONS}

This work extends and generalizes the recently proposed spectral bounds for the bond percolation to incorporate effects of triangles in the network. We propose a message passing algorithm for computing the size of percolating cluster considering triangles in the network, and demonstrate that the linearized version of this message passing algorithm, which uses the triangle-non-backtracking matrix, gives a tighter lower bounds than using other matrices, to the percolation transition on an arbitrary network. On synthetic networks generated by the clustered random graph 


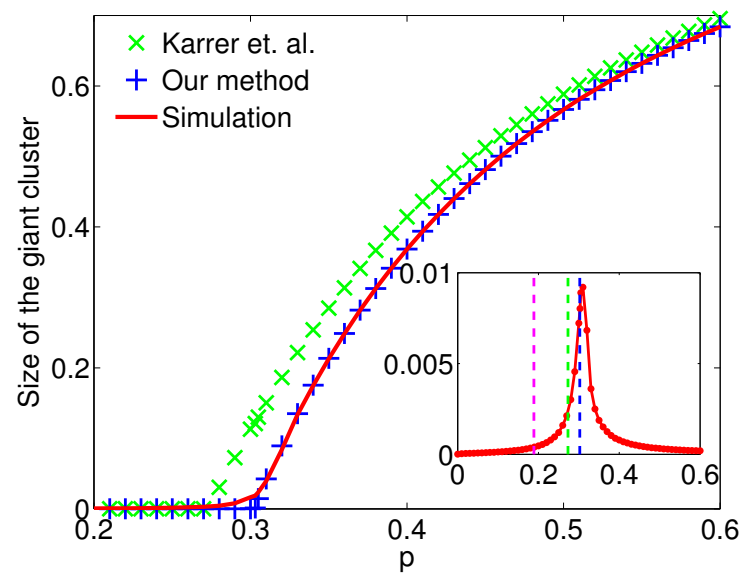

(a)

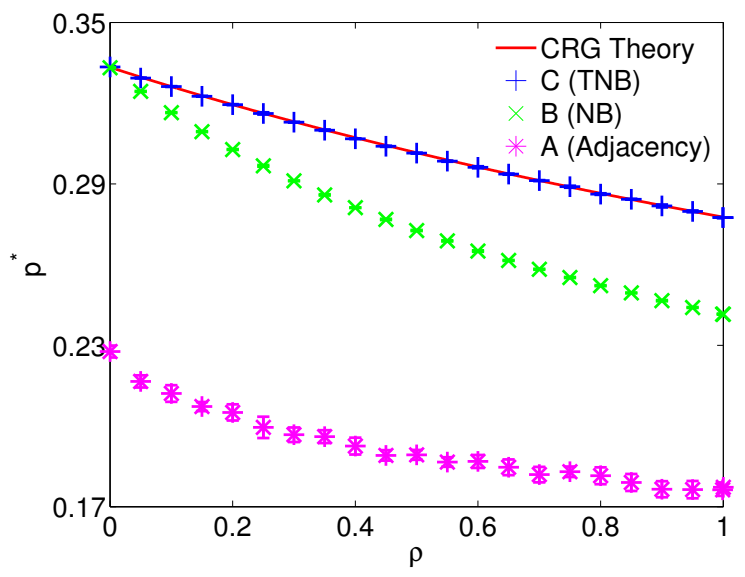

(b)

FIG. 3: (Color online) (a) Comparison of size of the giant clusters of simulations (averaged over 10 realizations), BP without triangles [20] and our message passing algorithm on a network generated by the clustered random graph model [28. The network has number of nodes $n=10^{5}$, average degree $c=3$, and fraction of edges belonging to triangles $\rho=0.5$. The inset shows the size of the second largest cluster in simulations which has a peak at the percolation transition. Three vertical lines, from left to right, in the inset are the estimates of percolation transition given using the adjacency matrix, the non-backtracking matrix and the triangle-non-backtracking matrix respectively. (b) From bottom to top, percolation transition given by the adjacency matrix $A$, the non-backtracking matrix $B$, the triangle-non-backtracking matrix $C$, and exact theory for $n \rightarrow \infty$ CRG networks in [28]. Networks have a Poisson degree distribution with average degree $c=3$. Number of nodes $n=10^{6}, \rho$ denotes fraction of edges belonging to triangles. Each point of simulation is averaged over 5 random realizations of edges' open-close states.

\begin{tabular}{c|ccccccc}
\hline Network & $n$ & $m$ & $\mathrm{CC}$ & $p_{A}^{*}$ & $p_{B}^{*}$ & $p_{C}^{*}$ & $p_{\text {Simu }}^{*}$ \\
\hline Karate club [29] & 34 & 78 & 0.571 & 0.149 & 0.189 & $0.197(1)$ & $0.229(5)$ \\
Les Miserables [30] & 77 & 254 & 0.573 & 0.0833 & 0.0930 & $0.09432(3)$ & $0.146(6)$ \\
Political books [31] & 105 & 441 & 0.488 & 0.0838 & 0.0941 & $0.0954(1)$ & $0.169(2)$ \\
Political Blogs [32] & 1490 & 16716 & 0.263 & 0.0135 & 0.0137 & $0.0138(0)$ & $0.0173(6)$ \\
Coauthorships [33] & 1589 & 2742 & 0.638 & 0.0526 & 0.0555 & $0.0558(0)$ & $0.45(1)$ \\
Protein [34] & 2115 & 2203 & 0.0594 & 0.133 & 0.198 & $0.206(1)$ & $0.31(1)$ \\
Power grid 35] & 4941 & 6594 & 0.08 & 0.134 & 0.161 & $0.166(1)$ & $0.659(7)$ \\
Collaboration [36] & 8361 & 15751 & 0.42 & 0.0435 & 0.0454 & $0.0456(0)$ & $0.121(3)$ \\
Email 37] & 36692 & 183831 & 0.497 & 0.00844 & 0.00866 & $0.00866(0)$ & 0.0104 \\
Gnutella [38] & 62586 & 147892 & 0.00546 & 0.0759 & 0.0871 & $0.0872(0)$ & 0.0967 \\
SBM [39] & 100000 & 149998 & $3.36 \times 10^{-5}$ & 0.229 & 0.3327 & $0.3328(0)$ & $0.341(1)$ \\
TSBM [unpublished] & 100000 & 99992 & 0.272 & 0.200 & 0.331 & $0.429(0)$ & $0.444(2)$ \\
\hline
\end{tabular}

TABLE I: Percolation transitions estimated using eigenvalues of the adjacency matrices $p_{A}^{*}$, non-backtracking matrices $p_{B}^{*}$, triangle-non-backtracking matrices $p_{C}^{*}$, and in direct simulations $p_{\text {simu }}^{*}$ which is estimated by finding the point where the second-largest cluster is greatest, by taking average over at least 1000 realizations of percolation process. Each $p_{C}^{*}$ value is averaged over10 different $\{\mathbb{T}, \mathbb{E}\}$ decompositions, numbers in parentheses indicate the error on the last digit. In each row, $n$ denotes the number of nodes, $m$ denotes the number of edges and $\mathrm{CC}$ indicates the clustering coefficient.

model we have shown that our estimate of the percolation transition on large networks agrees with the exact results for infinite networks. On real-world networks, we have shown that our estimate is always closer to the result of direct simulations than the estimates given by other spectral methods.

Incorporating effects of triangles is the first step towards understanding the effects of clustering to the percolation transitions. In principle our method can be generalized to include higher-order structures like quadrangles and pentagons. Without loss of generality, we can think about a structure like quadrangle. In this case we will be having a factor graph of 3 factors - single edges, triangles and quadrangles. By applying BP equations to this factor graph, then taking the linearization at the factorized fixed point, we will be arriving at a matrix that is a generalized version of our triangle-non-backtracking matrix, but considering quadrangles. Although the generalized matrix will be more and more complex when we consider higher and higher order structures, the gain is obvious: it gives a sequence of lower-bounds to the true percolation transition. We will put it in future study to test whether considering higher-order structures improves significantly the estimate of the transition for real-world networks. 
An interesting application of percolation is the network attack, or network dismantling problem. The problem asks to find the smallest set of nodes such that after their removal the size of the largest connected component is sub-extensive. The most efficient and effective methods for this problem are based on non-backtracking matrix [24, 25]. These methods essentially ask a fundamental question on how size of the LCC changes if one removes each node individually. It turns out that this question is difficult to answer by running direct simulations, but quite easy to study using spectral methods, which convert the problem to the shift of leading eigenvalue under perturbations of node removal. It would be interesting to study whether spectral dismantling methods based on the generalized non-backtracking matrix significantly outperforms the existing methods that based on the non-backtracking matrix.

Finally it worths noting that the $4 n \times 4 n$ form of the triangle non-backtracking matrix has a similar form to the non-backtracking operator defined for dynamic networks proposed in [40. It would be interesting to study the detailed relations between these two operators.

A $\mathrm{c}++$ implementaiton of our algorithm can be found at http://lib.itp.ac.cn/html/panzhang/perc.zip

\section{Acknowledgments}

We acknowledge helpful suggestions from the anonymous referee. Part of the computations have been carried out at the High Performance Computational Cluster of ITP, CAS.

\section{Appendix A: Proof of Lemma 1}

If the network contains no triangle, obviously the equality holds. For networks with triangles, observe that in any $\{\mathbb{T}, \mathbb{E}\}$ decomposition, all single edges are single edges in the original graph, thus the matrix $C$ corresponding to the decomposition has a smaller size than the matrix $B$. Note that the leading eigenvector $\left\{u_{i \rightarrow j}\right\}$, with eigenvalue $\lambda_{B}$, of the non-backtracking matrix [22]

$$
B_{i \rightarrow l, j \rightarrow k}=\delta(k, i)(1-\delta(j, l))
$$

satisfies

$$
u_{i \rightarrow l}=\frac{1}{\lambda_{B}} \sum_{j \in \partial i \backslash l} u_{j \rightarrow i}
$$

we can rewrite the last equation in a $\{\mathbb{T}, \mathbb{E}\}$ decomposition as

$$
\begin{aligned}
\lambda_{B} u_{i \rightarrow l} & =\sum_{j \in \partial^{-} i \backslash l} u_{j \rightarrow i}+\sum_{(i, j, k) \in \partial^{\Delta} i}\left(u_{k \rightarrow i}+u_{j \rightarrow i}\right) \\
& =\sum_{j \in \partial^{-} i \backslash l} u_{j \rightarrow i}+\sum_{(i, j, k) \in \partial^{\Delta} i} u_{(j, k) \rightarrow i} .
\end{aligned}
$$

Here $u_{(j, k) \rightarrow i}$ represents the sum of messages from two other end-points in the triangle $(i, j, k)$, and it can be further evaluated as

$$
\begin{aligned}
\lambda_{B} u_{(j, k) \rightarrow i} & \left.=\sum_{j^{\prime} \in \partial^{-} j} u_{j^{\prime} \rightarrow j}+\sum_{(j, r, s) \in \partial^{\Delta} \backslash(i, j, k)} u_{(r, s) \rightarrow j}+\sum_{k^{\prime} \in \partial^{-} k} u_{k^{\prime} \rightarrow k}+\sum_{(k, r, s) \in \partial^{\Delta} k \backslash(i, j, k)} u_{(r, s) \rightarrow k}+u_{j \rightarrow k}+u_{k \rightarrow j} u_{(r, s) \rightarrow k} u_{(r, s) \rightarrow j}+\sum_{k^{\prime} \in \partial^{-} k} u_{k^{\prime} \rightarrow k}+\sum_{(k, r, s) \in \partial^{\Delta} k \backslash(i, j, k)} u_{j^{\prime} \rightarrow j}+\sum_{(j, r, s) \in \partial^{\Delta} \backslash \backslash(i, j, k)} u_{(r, s) \rightarrow j}+u_{i \rightarrow j}+\sum_{k^{\prime} \in \partial^{-} k} u_{k^{\prime} \rightarrow k}+\sum_{(k, r, s) \in \partial^{\Delta} k \backslash(i, j, k)} u_{(r, s) \rightarrow k}+u_{i \rightarrow k}\right) \\
& +\frac{1}{\lambda_{B}} \sum_{j^{\prime} \in \partial^{-} j} u_{j^{\prime} \rightarrow j}+\sum_{(j, r, s) \in \partial^{\Delta} j \backslash(i, j, k)} \sum_{\left(1+\frac{1}{\lambda_{B}}\right)}\left(\sum_{j^{\prime} \in \partial^{-} j} u_{j^{\prime} \rightarrow j}+\sum_{(j, r, s) \in \partial^{\Delta} j \backslash(i, j, k)} u_{(r, s) \rightarrow j} u_{(\mathrm{A} 2)} u_{(r, s) \rightarrow k}\right)+\frac{1}{\lambda_{B}}\left(u_{i \rightarrow k}+u_{i \rightarrow j)} .\right.
\end{aligned}
$$


Then we define a new matrix $B^{\prime}$ with size $\left(n^{-}+n^{\Delta}\right) \times\left(n^{-}+n^{\Delta}\right)$, as

$$
B_{x \rightarrow i, z \rightarrow w}^{\prime}=p \mathbb{1}_{x \notin \mathbb{T}} \delta(x, w)(1-\delta(z, i))+p\left(1+\frac{1}{\lambda_{B}}\right) \mathbb{1}_{x \in \mathbb{T}} \mathbb{1}_{w \in x \backslash i} \mathbb{1}_{z \notin x}+\frac{p}{\lambda_{B}} \mathbb{1}_{x \in \mathbb{T}} \mathbb{1}_{w \in x \backslash i} \delta(z, i) .
$$

We can see that the leading eigenvalue of $p B$ and $B^{\prime}$ are the same.

Since matrix $C$ is defined as (see main text):

$$
C_{x \rightarrow i, z \rightarrow w}=p \mathbb{1}_{x \notin \mathbb{T}} \delta(x, w)(1-\delta(z, i))+q \mathbb{1}_{x \in \mathbb{T}} \mathbb{1}_{w \in x \backslash i} \mathbb{1}_{z \notin x} .
$$

It is easy to see that $B^{\prime}$ and $C$ have the same size. With $q=1+p^{2}-p^{3}$ we have

$$
B_{x \rightarrow i, z \rightarrow w}^{\prime}-C_{x \rightarrow i, z \rightarrow w}=\left(\frac{p}{\lambda_{B}}-p^{2}+p^{3}\right) \mathbb{1}_{x \in \mathbb{T}} \mathbb{1}_{w \in x \backslash i} \mathbb{1}_{z \notin x}+\frac{p}{\lambda_{B}} \mathbb{1}_{x \in \mathbb{T}} \mathbb{1}_{w \in x \backslash i} \delta(z, i) .
$$

As $p \geq 0$, the last term in the right hand side of last equation is always non-negative. We can also see that together with many other conditions, under condition $p \lambda_{B} \leq 1$, we have

$$
\frac{p}{\lambda_{B}}=\frac{p^{2}}{p \lambda_{B}} \geq p^{2} \geq p^{2}-p^{3},
$$

thus every element of $B^{\prime}-C$ is non-negative. By applying the Collatz-Wielandt theorem, the leading eigenvalue of $B^{\prime}$ satisfies

$$
p \lambda_{B} \geq \min _{x \rightarrow y, v_{x \rightarrow y} \neq 0} \frac{\left(B^{\prime} v\right)_{x \rightarrow y}}{v_{x \rightarrow y}} .
$$

where $v_{x \rightarrow y}$ can be any real vector with length $n^{-}+n^{\Delta}$. If we choose $v$ to be the leading eigenvector of $C$, whose elements are non-negative, then under $p \lambda_{B} \leq 1$, we have

$$
p \lambda_{B} \geq \min _{x \rightarrow y, v_{x \rightarrow y} \neq 0}\left(\frac{[C v]_{x \rightarrow y}}{v_{x \rightarrow y}}+\frac{\left[\left(B^{\prime}-C\right) v\right]_{x \rightarrow y}}{v_{x \rightarrow y}}\right) \geq \lambda_{C} .
$$

The proof is complete.

\section{Appendix B: Proof of Theorem 4}

Eigenvector $\epsilon$ associated with a non-zero eigenvalue $\lambda$ of matrix $C$ satisfies

$$
\begin{aligned}
& \lambda \epsilon^{i \rightarrow j}=p \sum_{i^{\prime} \in \partial i^{-} \backslash j} \epsilon^{i^{\prime} \rightarrow i}+p \sum_{a \in \partial^{\Delta} i} \epsilon^{a \rightarrow i} \\
& \lambda \epsilon^{a \rightarrow i}=q \sum_{j^{\prime} \in \partial j^{-}} \epsilon^{j^{\prime} \rightarrow j}+q \sum_{b \in \partial^{\Delta} j \backslash a} \epsilon^{b \rightarrow j}+q \sum_{k^{\prime} \in \partial k^{-}} \epsilon^{k^{\prime} \rightarrow k}+q \sum_{f \in \partial^{\Delta} k \backslash a} \epsilon^{f \rightarrow k},
\end{aligned}
$$

where triangle $a=(i, j, k)$.

Let us define sum of incoming messages and outgoing messages for node $i$ along edges connected to node $i$ as

$$
\epsilon_{\mathrm{in}}^{i-}=\sum_{i^{\prime} \in \partial^{-} i} \epsilon^{i^{\prime} \rightarrow i} \quad \epsilon_{\text {out }}^{i-}=\sum_{i^{\prime} \in \partial^{-} i} \epsilon^{i \rightarrow i^{\prime}} .
$$

Sum of incoming messages and outgoing messages for node $i$ along triangles connected to node $i$ can be defined in a similar way:

$$
\epsilon_{\mathrm{in}}^{i \Delta}=\sum_{a \in \partial^{\Delta} i} \epsilon^{a \rightarrow i} \quad \epsilon_{\text {out }}^{i \Delta}=\sum_{a \in \partial^{\Delta}} \sum_{j \in a \backslash i} \epsilon^{a \rightarrow j}
$$


Inserting Eq. (B1) into Eq. (B4) we have an expression for sum of incoming messages along single edges to node $i$ :

$$
\begin{aligned}
\lambda \epsilon_{\mathrm{in}}^{i-} & =\lambda \sum_{i^{\prime} \in \partial^{-} i} \epsilon^{i^{\prime} \rightarrow i} \\
& =p \sum_{i^{\prime} \in \partial^{-} i}\left(\sum_{i^{\prime \prime} \in \partial i^{\prime}-\backslash i} \epsilon^{i^{\prime \prime} \rightarrow i^{\prime}}+\sum_{a \in \partial^{\Delta} i^{\prime}} \epsilon^{a \rightarrow i^{\prime}}\right) \\
& =p \sum_{i^{\prime} \in \partial^{-} i}\left(\sum_{i^{\prime \prime} \in \partial i^{\prime-}} \epsilon^{i^{\prime \prime} \rightarrow i^{\prime}}+\sum_{a \in \partial^{\Delta} i^{\prime}} \epsilon^{a \rightarrow i^{\prime}}-\epsilon^{i \rightarrow i^{\prime}}\right) \\
& =p \sum_{i^{\prime} \in \partial^{-} i}\left(\epsilon_{\mathrm{in}}^{i^{\prime}-}+\epsilon_{\mathrm{in}}^{i^{\prime} \Delta}\right)-p \epsilon_{\mathrm{out}}^{i-},
\end{aligned}
$$

and also for sum of outgoing messages along single edges from node $i$ :

$$
\begin{aligned}
\lambda \epsilon_{\mathrm{out}}^{i-} & =\lambda \sum_{i^{\prime} \in \partial^{-} i} \epsilon^{i \rightarrow i^{\prime}} \\
& =p \sum_{i^{\prime} \in \partial^{-} i}\left(\sum_{i^{\prime \prime} \in \partial i^{-} \backslash i^{\prime}} \epsilon^{i^{\prime \prime} \rightarrow i}+\sum_{a \in \partial^{\Delta}} \epsilon^{a \rightarrow i}\right) \\
& =p \sum_{i^{\prime} \in \partial^{-} i}\left(\sum_{i^{\prime \prime} \in \partial i^{-}} \epsilon^{i^{\prime \prime} \rightarrow i}+\sum_{a \in \partial^{\Delta}} \epsilon^{a \rightarrow i}-\epsilon^{i^{\prime} \rightarrow i}\right) \\
& =p\left[\left(d_{i}^{-}-1\right) \epsilon_{\mathrm{in}}^{i-}+d_{i}^{-} \epsilon_{\mathrm{in}}^{i \Delta}\right] .
\end{aligned}
$$

Similarly, sum of messages coming from a triangle to node $i$ can be evaluated as

$$
\begin{aligned}
\lambda \epsilon_{\mathrm{in}}^{i \Delta} & =\lambda \sum_{a \in \partial^{\Delta} i} \epsilon^{a \rightarrow i} \\
& =q \sum_{a \in \partial^{\Delta}} \sum_{i}\left(\sum_{j \in a \backslash i} \epsilon^{j^{\prime} \rightarrow j}+\sum_{b \in \partial j^{\prime} \backslash a} \epsilon^{b \rightarrow j}\right) \\
& =q \sum_{a \in \partial^{\Delta}} \sum_{i}\left(\sum_{j \in a \backslash i} \epsilon_{j^{\prime} \in \partial j-\backslash a}^{j^{\prime} \rightarrow j}+\sum_{b \in \partial j^{\Delta}} \epsilon^{b \rightarrow j}-\epsilon^{a \rightarrow i}\right) \\
& =q \sum_{a \in \partial^{\Delta}} \sum_{i \in a \backslash i}\left(\epsilon_{\mathrm{in}}^{j-}+\epsilon_{\mathrm{in}}^{j \Delta}\right)-q \epsilon_{\mathrm{out}}^{i \Delta},
\end{aligned}
$$

where $j \in a$ means $j$ is one end point of triangle $a$. Sum of outgoing message going to triangles from node $i$ are written as

$$
\begin{aligned}
\lambda \epsilon_{\mathrm{out}}^{i \Delta} & =\lambda \sum_{a \in \partial^{\Delta}} \sum_{i} \epsilon_{j \in a \backslash i}^{a \rightarrow j} \\
& =q \sum_{a \in \partial^{\Delta}} \sum_{i} \sum_{j \in a \backslash i}\left(\epsilon_{\mathrm{in}}^{k-}+\epsilon_{\mathrm{in}}^{k \Delta}-\epsilon^{a \rightarrow k}\right) \\
& =2 q\left(d_{i}^{\Delta} \epsilon_{\mathrm{in}}^{i-}+d_{i}^{\Delta} \epsilon_{\mathrm{in}}^{i \Delta}-\epsilon_{\mathrm{in}}^{i \Delta}\right)+q \sum_{a \in \partial^{\Delta}} \sum_{j \in a \backslash i}\left(\epsilon_{\mathrm{in}}^{j-}+\epsilon_{\mathrm{in}}^{j \Delta}-\epsilon^{a \rightarrow i}\right) \\
& =2 q\left[d_{i}^{\Delta} \epsilon_{\mathrm{in}}^{i-}+\left(d_{i}^{\Delta}-1\right) \epsilon_{\mathrm{in}}^{i \Delta}\right]+q \sum_{a \in \partial^{\Delta}} \sum_{j \in a \backslash i}\left(\epsilon_{\mathrm{in}}^{j-}+\epsilon_{\mathrm{in}}^{j \Delta}\right)-q \epsilon_{\mathrm{out}}^{i \Delta}
\end{aligned}
$$

Thus if we let $\epsilon_{\mathrm{in}}^{-}=\left\{\epsilon_{\mathrm{in}}^{i-}, i \in\{1, \ldots, n\}\right\}, \epsilon_{\mathrm{in}}^{\Delta}=\left\{\epsilon_{\mathrm{in}}^{i \Delta}, i \in\{1, \ldots, n\}\right\}, \epsilon_{\text {out }}^{-}=\left\{\epsilon_{\text {out }}^{i-}, i \in\{1, \ldots, n\}\right\}, \epsilon_{\text {out }}^{\Delta}=\left\{\epsilon_{\text {out }}^{i \Delta}, i \in\right.$ $\{1, \ldots, n\}\}$, then in a matrix form, above equations can be written as

$$
\lambda\left(\begin{array}{c}
\epsilon_{\text {in }}^{-} \\
\epsilon_{\text {out }}^{-} \\
\epsilon_{\text {in }}^{\Delta} \\
\epsilon_{\text {out }}^{\Delta}
\end{array}\right)=C^{\prime}\left(\begin{array}{c}
\epsilon_{\text {in }}^{-} \\
\epsilon_{\text {out }}^{-} \\
\epsilon_{\text {in }}^{\Delta} \\
\epsilon_{\text {out }}^{\Delta}
\end{array}\right)
$$


with

$$
C^{\prime}=\left(\begin{array}{cccc}
p \mathbb{A}^{-} & -p \mathbb{I} & p \mathbb{A}^{-} & 0 \\
p\left(\mathbb{D}^{-}-\mathbb{I}\right) & 0 & p \mathbb{D}^{-} & 0 \\
q \mathbb{A}^{\Delta} & 0 & q \mathbb{A}^{\Delta} & -q \mathbb{I} \\
2 q \mathbb{D}^{\Delta}+q \mathbb{A}^{\Delta} & 0 & 2 q\left(\mathbb{D}^{\Delta}-\mathbb{I}\right)+q \mathbb{A}^{\Delta} & -q \mathbb{I}
\end{array}\right)
$$

where $\mathbb{I}$ denotes the $n$-dimensional identity matrix, $\mathbb{A}^{-}$is the adjacency matrix of nodes connected by single edges; $\mathbb{D}^{-}$ is the diagonal matrix of single-edge-degrees; $\mathbb{A}^{\Delta}$ is the adjacency matrix of nodes connected by triangles; $\mathbb{D}^{\Delta}$ is the diagonal matrix of triangle-degrees. That is

$$
\begin{array}{ll}
\mathbb{A}_{i, j}^{-}= \begin{cases}1, \text { if }(i, j) \in \mathbb{E} \\
0, \text { other wise }\end{cases} & \mathbb{A}_{i, j}^{\Delta}=\left\{\begin{array}{l}
1, \text { if } \exists(i, j, l) \in \mathbb{T} \\
0, \text { other wise }
\end{array}\right. \\
\mathbb{D}_{i, i}^{-}=d_{i}^{-}=\sum_{k} \mathbb{A}_{i k}^{-} & \mathbb{D}_{i, i}^{\Delta}=d_{i}^{\Delta}=\frac{1}{2} \sum_{k} \mathbb{A}_{i k}^{\Delta} .
\end{array}
$$

Thus we can see that $\lambda$ is both an eigenvalue of matrix $C$ and matrix $C^{\prime}$.

[1] M. E. J. Newman, Phys. Rev. E 66, 016128 (2002), URL http://link.aps.org/doi/10.1103/PhysRevE.66.016128

[2] R. Cohen, K. Erez, D. ben Avraham, and S. Havlin, Phys. Rev. Lett. 85, 4626 (2000), URL http://link. aps.org/doi/10 1103/PhysRevLett.85.4626

[3] J. Machta, Phys. Rev. Lett. 66, 169 (1991), URL http://link.aps.org/doi/10.1103/PhysRevLett.66.169.

[4] S. Bondt, L. Froyen, and A. Deruyttere, Journal of Materials Science 27, 1983 (1992), ISSN 1573-4803, URL http: //dx.doi.org/10.1007/BF01107228

[5] C. L. Henley, Phys. Rev. Lett. 71, 2741 (1993), URL http://link.aps.org/doi/10.1103/PhysRevLett.71.2741

[6] R. Albert, H. Jeong, and A.-L. Barabási, nature 406, 378 (2000).

[7] S. Kirkpatrick, Rev. Mod. Phys. 45, 574 (1973), URL http://link.aps.org/doi/10.1103/RevModPhys.45.574.

[8] V. Ambegaokar, B. I. Halperin, and J. S. Langer, Phys. Rev. B 4, 2612 (1971), URL http://link.aps.org/doi/10.1103/ PhysRevB.4.2612.

[9] D. J. Bergman and Y. Imry, Phys. Rev. Lett. 39, 1222 (1977), URL http://link.aps.org/doi/10.1103/PhysRevLett.39 1222

[10] P. Holme, B. J. Kim, C. N. Yoon, and S. K. Han, Phys. Rev. E 65, 056109 (2002), URL http://link.aps.org/doi/10 1103/PhysRevE.65.056109

[11] A. V. Goltsev, S. N. Dorogovtsev, and J. F. F. Mendes, Phys. Rev. E 78, 051105 (2008), URL http://link.aps.org/doi/ 10.1103/PhysRevE.78.051105.

[12] D. S. Callaway, M. E. J. Newman, S. H. Strogatz, and D. J. Watts, Phys. Rev. Lett. 85, 5468 (2000), URL http: //link.aps.org/doi/10.1103/PhysRevLett.85.5468.

[13] J. P. Gleeson, Phys. Rev. E 80, 036107 (2009), URL http://link.aps.org/doi/10.1103/PhysRevE.80.036107.

[14] J. P. Gleeson, S. Melnik, and A. Hackett, Phys. Rev. E 81, 066114 (2010), URL http://link.aps.org/doi/10.1103/ PhysRevE.81.066114.

[15] J. C. Miller, Physical Review E 80, 020901 (2009).

[16] M. A. Serrano and M. Boguñá, Phys. Rev. Lett. 97, 088701 (2006), URL http://link.aps.org/doi/10.1103/PhysRevLett 97.088701.

[17] M. A. Serrano and M. Boguñá, Phys. Rev. E 74, 056115 (2006), URL http://link.aps.org/doi/10.1103/PhysRevE.74 056115 .

[18] B. Bollobás, C. Borgs, J. Chayes, O. Riordan, et al., The Annals of Probability 38, 150 (2010).

[19] K. E. Hamilton and L. P. Pryadko, Phys. Rev. Lett. 113, 208701 (2014), URL http://link.aps.org/doi/10.1103/ PhysRevLett.113.208701.

[20] B. Karrer, M. E. J. Newman, and L. Zdeborová, Phys. Rev. Lett. 113, 208702 (2014), URL http://link.aps.org/doi/10 1103/PhysRevLett.113.208702

[21] K.-i. Hashimoto, Advanced Studies in Pure Mathematics 15, 211 (1989).

[22] F. Krzakala, C. Moore, E. Mossel, J. Neeman, A. Sly, L. Zdeborová, and P. Zhang, Proc. Natl. Acad. Sci. USA 110, 20935 (2013), http://www.pnas.org/content/110/52/20935.full.pdf +html.

[23] P. Zhang, Physical Review E 91, 042120 (2015).

[24] F. Morone, and H. Makse, Nature 524, 65 (2015),

[25] L. Zdeborová, P. Zhang, and H. Zhou, Scientific Reports 6, 37954 (2016).

[26] J. Yedidia, W. Freeman, and Y. Weiss, in International Joint Conference on Artificial Intelligence (IJCAI) (2001).

[27] R. Monasson, R. Zecchina, S. Kirkpatrick, B. Selman, and L. Troyansky, Nature 400, 133 (1999).

[28] M. E. Newman, Physical review letters 103, 058701 (2009). 
[29] W. W. Zachary, Journal of anthropological research pp. 452-473 (1977).

[30] D. E. Knuth, The Stanford GraphBase: A Platform for Combinatorial Computing (Addison-Wesley, Reading, MA, 1993).

[31] http://www.orgnet.com/.

[32] L. A. Adamic and N. Glance, in Proceedings of the 3rd international workshop on Link discovery (ACM, 2005$)$, pp. 36-43.

[33] M. E. J. Newman, Phys. Rev. E 74, 036104 (2006), URL http://link.aps.org/doi/10.1103/PhysRevE.74.036104

[34] A. B. Z. N. Hawoong Jeong, S PMason, 411, 41 (2001).

[35] D. Watts and S. Strogatz, Nature(London) 393, 440 (1998).

[36] M. E. Newman, Proceedings of the National Academy of Sciences 98, 404 (2001).

[37] J. Leskovec, K. J. Lang, A. Dasgupta, and M. W. Mahoney, Internet Mathematics 6, 29 (2009).

[38] R. Matei, A. Iamnitchi, and I. Foster, Internet Computing, IEEE 6, 50 (2002).

[39] P. W. Holland, K. B. Laskey, and S. Leinhardt, Social networks 5, 109 (1983).

[40] A. Ghasemian, P. Zhang, A. Clauset, C. Moore, and L. Peel, Phys. Rev. X 6, 031005 (2016).

[41] F. Krzakala, A. Montanari, F. Ricci-Tersenghi, G. Semerjian and L. Zdeborová, Proc. Natl. Acad. Sci. USA 104, 10318 (2007).

[42] A. Decelle, F. Krzakala, C. Moore and L. Zdeborová, Phys. Rev. Lett. 107, 065701 (2011). 